SHORT REPORT

\title{
Reversible bilateral internuclear ophthalmoplegia associated with FK506
}

\author{
M M Lai, J B Kerrison, N R Miller
}

J Neurol Neurosurg Psychiatry 2004;75:776-778. doi: 10.1136/jnnp.2003.027375

\begin{abstract}
A 50 year old man developed tonic-clonic seizures while receiving cyclosporin $\mathrm{A}$ after orthotopic cardiac transplant. The seizures resolved after cessation of cyclosporin $A$. Thirteen months later, he developed diplopia from bilateral internuclear ophthalmoplegia while receiving intravenous FK506. A temporal association was found between his symptoms and the serum FK506 concentrations. Withdrawal of the intravenous FK506 led to prompt resolution of the bilateral internuclear ophthalmoplegia.
\end{abstract}

$\mathrm{F}$ K506 (tacrolimus) and cyclosporin A (CsA) are immunosuppressive drugs used to prevent solid organ or bone marrow transplant rejections. Both drugs inhibit $\mathrm{T}$ cell activation via the common mechanism of calcineurin phosphatase inhibition. ${ }^{1}$ Neurological side effects have been reported in patients receiving either FK506 or CsA. ${ }^{23}$ We report on a patient with reversible bilateral internuclear ophthalmoplegia (INO) in the setting of high dose FK506 therapy who also had seizures secondary to CyA use.

\section{CASE REPORT}

A 50 year old man underwent an orthotopic cardiac transplant for ischaemic cardiomyopathy 13 months before his current hospitalisation. After this surgery, the patient was treated with CsA, prednisone, mycophenylate mofetil, and azathioprine. During this period, the patient developed tonicclonic seizures. A lumbar puncture and head computed tomography performed at the time were reportedly normal. Based on evaluations performed by the hospital neurology consultation service, his seizures were attributed to CsA toxicity. The seizures resolved after withdrawal of CsA.

About three months before his current hospitalisation, the patient developed evidence of graft rejection and was given FK506 taken orally at a dose of $4 \mathrm{mg} /$ day, which he tolerated despite mild headaches. Despite treatment with FK506, the patient experienced progressive rejection of his graft, characterised clinically by worsening left ventricular function. He therefore was admitted to the hospital for placement of a left ventricular assist device. At this time, he was also administered intravenous FK506 with the goal of obtaining a drug concentration in the serum between 10 and $20 \mathrm{ng} / \mathrm{ml}$. His treatment drugs at the time of admission also included prednisone ( $5 \mathrm{mg}$ twice daily orally), azathioprine $(50 \mathrm{mg}$ once daily orally), lisinopril ( $15 \mathrm{mg}$ once daily orally), digoxin (0.125 mg once daily orally), and levothyroxine ( $100 \mu \mathrm{g}$ once daily orally).

Three days after starting intravenous FK506, the patient developed worsening headache and horizontal diplopia. An ophthalmology consultation was obtained the following day. On examination, his corrected visual acuity was 20/30 in each eye. Pupils were isocoric and normally reactive with no relative afferent pupillary defect. Intraocular pressure measurements, colour vision, and visual fields were normal in both eyes. In primary position, the patient had an exotropia of 28 prism diopters, which increased on both right and left horizontal gaze but not on upward or downward gaze (fig 1A). On attempted horizontal gaze, there was normal abduction but decreased adduction associated with reduced saccadic velocities of the adducting eye and jerk horizontal nystagmus of the abducting eye with the fast phase in the direction of gaze. No convergence could be elicited. The anterior segment examination and a dilated ophthalmoscopic examination were both within normal limits in each eye.

A diagnosis of bilateral INO was made. The patient's vital signs and serum electrolytes were stable, and several sets of blood cultures taken around this time were unremarkable. However, his serum FK506 concentration had risen from $5.2 \mathrm{ng} / \mathrm{ml}$ on the day intravenous FK506 was started (day l) to $18.7 \mathrm{ng} / \mathrm{ml}$ on the day his symptoms began (day 4 ), and continued to rise to $21.4 \mathrm{ng} / \mathrm{ml}$ on the day of his ophthalmic examination (day 5) (table 1). FK506 induced neurotoxicity was suspected. Magnetic resonance imaging (MRI) of the brain was recommended; however, the MRI could not be performed because the patient harboured magnetic metal in his ventricular assist device. Accordingly, the decision was made to stop the intravenous FK506 (day 5). Within 48 hours after stopping FK506, the patient had experienced complete resolution of his headache and diplopia. Repeat examination performed two days after stopping FK506 (day 7) showed complete resolution of the bilateral INO with normal ocular motility and alignment and normal horizontal saccadic velocities of both eyes on both right and left gaze (fig 1B). A repeat serum FK506 concentration at this time was $13.3 \mathrm{ng} / \mathrm{ml}$ (table 1 ).

\section{DISCUSSION}

FK506 and CsA are potent immunosuppressive agents used in a variety of clinical settings, including the treatment of autoimmune disorders and the prevention of graft versus host disease after bone marrow or organ transplantation. Although structurally dissimilar, both drugs are microbial metabolites originally isolated from soil samples, and both selectively inhibit the same cellular target, the calcium sensitive protein phosphatase calcineurin. ${ }^{1}$ Calcineurin's phosphatase activity is necessary for the production of several molecules involved in $\mathrm{T}$ cell activation, including interleukin 2. Therefore, both FK506 and CsA suppress T cell activation by inhibiting calcineurin.

The most common side effects of FK506 and CsA are renal failure and hypertension. However, neurological toxicities may occur in patients receiving either drug. ${ }^{23}$ Neurological manifestations of FK506 or CsA toxicity range from mild symptoms such as headache, paresthesia, and tremor, to major complications including seizures, cortical blindness,

Abbreviations: $M R I$, magnetic resonance imaging; $C s A$, cyclosporin $A$; INO, internuclear ophthalmoplegia 
A
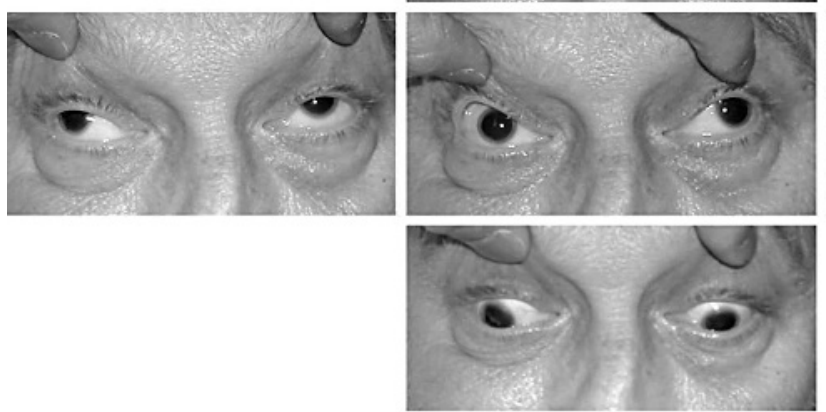

B
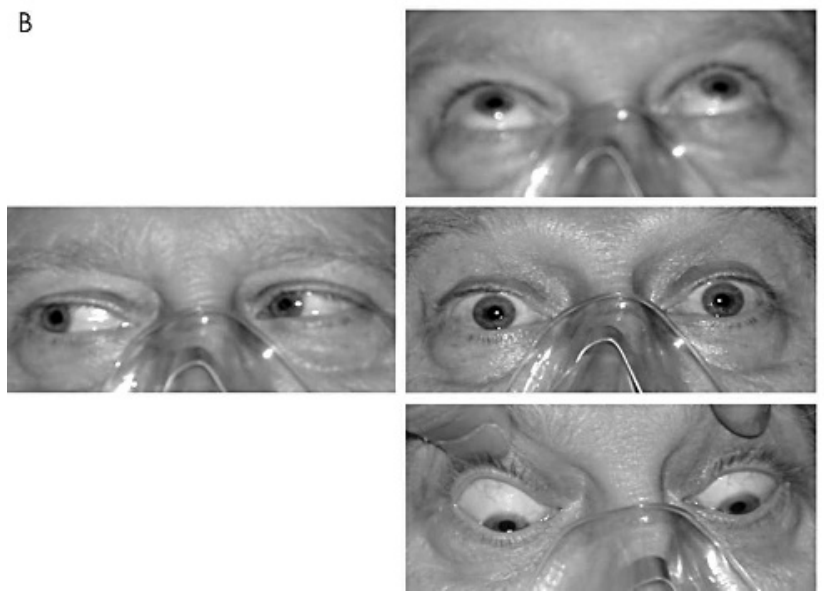

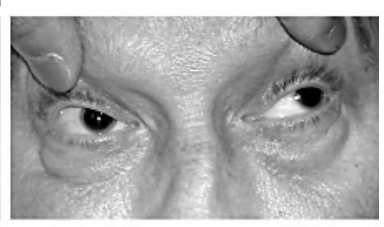

Figure 1 (A) Ocular motility examination discloses bilateral internuclear ophthalmoplegia three days after starting high dose intravenous FK506 therapy. (The patient's pupils were pharmacologically dilated.) (B) Resolution of ocular symptoms 48 hours after FK506 was withdrawn. encephalopathy, and coma. Tremor is the most common neurological symptom attributable to either drug and has been reported in up to $40 \%$ of patients receiving CsA and up to $54 \%$ of patients receiving FK506. ${ }^{23}$ About 5\% of patients receiving either CsA or FK506 develop one of the major neurological complications listed above. ${ }^{2}$ MRI studies in these patients typically show cerebral white matter abnormalities localised to the posterior cerebral hemispheres. ${ }^{3}$ The neurological symptoms, along with MRI findings, are usually reversible, resolving after withdrawal or reduction of the drug; however, cases of permanent neurological dysfunction have been reported. ${ }^{4}$

The precise mechanism of FK506 or CsA induced neurotoxicity is not known. Given that the two drugs have similar profiles of neurotoxicity ${ }^{6}$ and share a common cellular target, it is tempting to speculate that the inhibition of calcineurin is responsible for most if not all of the neurological symptoms associated with the use of CsA or FK506. Calcineurin is enriched in the central nervous system and comprises over $1 \%$ of the total protein in brain. ${ }^{7}$ Changes in calcineurin activity in the brain affect a wide range of neuronal processes, including intracellular signalling, apoptosis, release of neurotransmitters, and the recycling of neurotransmitter vesicles. ${ }^{8}{ }^{9}$ Inhibition of calcineurin also changes sympathetic outflow, ${ }^{10}$ which can contribute to some of the neurological symptoms by either direct cellular toxicity or changes in brain tissue perfusion.

INO results from damage to the medial longitudinal fasciculus, manifesting as variable weakness of adduction ipsilateral to the side of the lesion associated with reduced saccadic velocities of the adducting eye on horizontal gaze and horizontal jerk nystagmus in the abducting eye. Lesions that affect the medial longitudinal fasciculus on both sides cause bilateral INO. The presence of exotropia in the setting of bilateral INO constitutes the wall eyed bilateral INO syndrome, and implicates possible concomitant involvement of the medial rectus subnuclei. INO with absence of convergence is termed Cogan's anterior INO and implies the presence of a mesencephalic lesion. This patient had a wall eyed bilateral INO syndrome with inability to converge, suggesting that the causative lesion was in the mesencephalon and involved the medical rectus subnuclei as well as the

\begin{tabular}{|c|c|c|c|c|c|c|c|}
\hline Hospitalisation day & 1 & 2 & 3 & 4 & 5 & 6 & 7 \\
\hline $\begin{array}{l}\text { Serum FK506 concentration } \\
\text { (ng/ml) }\end{array}$ & 5.2 & 5.8 & 7.7 & 18.7 & 21.4 & 23.6 & 13.3 \\
\hline
\end{tabular}


medial longitudinal fasciculus, although it must be emphasised that many patients with INOs caused by pontine damage also are unable to converge. The differential diagnosis of bilateral INO includes multiple sclerosis, brain stem infarction, brain stem mass, infection, metabolic disorders, and drug intoxications. Although a MRI could not be performed in this patient secondary to the presence of metallic foreign body, we believe the most probable cause of the patient's bilateral INO was a toxic reaction caused by FK506. In support of this contention, this patient's laboratory studies did not reveal an infectious or metabolic aetiology. Furthermore, the rapid resolution of the ocular motor deficit after withdrawal of FK506 is incompatible with a demyelinating lesion, brain mass, or brain stem infarction.

Our case is unusual for several reasons. Firstly, to our knowledge, this is the first case of bilateral INO attributable to FK506 toxicity. Oliverio et al described a patient who developed bilateral sixth nerve pareses and INO while receiving FK506, although it was not clear from the clinical description if the INO was unilateral or bilateral. ${ }^{11}$ Secondly, the lesion in our patient clinically localises to the brain stem, whereas most reported cases of FK506 neurotoxicity show abnormalities in the cerebral hemispheres. Diplopia from unilateral or bilateral sixth nerve pareses has been reported in a few patients receiving $\mathrm{CsA}^{12}$ but the case reported by Oliverio et al is the only other case of reversible FK506 neurotoxicity isolated to the brain stem. ${ }^{11}$ Thirdly, our patient developed neurotoxic symptoms while his serum FK506 concentration was still within the intended therapeutic range of 10 to $20 \mathrm{ng} / \mathrm{ml}$, but lowering the FK506 concentration to the lower part of this therapeutic range was sufficient for the complete resolution of his symptoms. A report by McMaster et al found that serum FK506 concentrations of about $20 \mathrm{ng} /$ $\mathrm{ml}$ were commonly associated with neurotoxicity ${ }^{13}$; therefore, therapeutic monitoring is important in the management of FK506 associated neurotoxicity. Fourthly, our patient developed neurological symptoms and signs from both CsA and FK506. Although some patients have experienced improvement in neurological symptoms when converted from CsA to FK506, many patients showed no improvement. ${ }^{14}$ As discussed previously, calcineurin inhibition may be a common mechanism for neurotoxicities secondary to both FK506 and CsA. We therefore recommend that patients with a history of neurotoxicity caused by either CsA or FK506 be monitored closely for the development of new neurological symptoms when receiving another calcineurin inhibitor.

\section{Authors' affiliations}

M M Lai, J B Kerrison, N R Miller, Wilmer Eye Institute, Johns Hopkins Hospital, Baltimore, USA

Competing interests: none declared

Correspondence to: $\operatorname{Dr} N$ R Miller, Wilmer Eye Institute, Johns Hopkins Hospital, 600 North Wolfe Street, Baltimore, MD 21287, USA; nrmiller@ihmi.edu

Received 1 September 2003

Accepted in revised form 14 October 2003

\section{REFERENCES}

1 Ho S, Clipstone N, Timmermann L, et al. The mechanism of action of cyclosporin A and FK506. Clin Immunol Immunopathol 1996;80:S40-5

2 Wechstein WO. Neurotoxicity of calcineurin inhibitors: impact and clinical management. Transpl Int 2000;13:313-26.

3 Gijtenbeek JM, van den Bent MJ, Vecht CJ. Cyclosporine neurotoxicity: a review. J Neurol 1999;246:339-46.

4 Misawa A, Takeuchi Y, Hibi S, et al. FK506-induced intractable leukoencephalopathy following allogeneic bone marrow transplantation. Bone Marrow Transplant 2000;25:331-4.

5 Eidelman BH, Abu-Elmagd K, Wilson J, et al. Neurologic complications of FK 506. Transplant Proc 1991;23:3175-8.

6 Woo M, Przepiorka D, Ippoliti C, et al. Toxicities of tacrolimus and cyclosporin A after allogeneic blood stem cell transplantation. Bone Marrow Transplant 1997; 20:1095-8.

7 Yakel JL. Calcineurin regulation of synaptic function: from ion channels to transmitter release and gene transcription. Trends Pharmacol Sci 1997; 18:124-34.

8 Snyder SH, Lai MM, Burnett PE. Immunophilins in the nervous system. Neuron 1998;21:283-94.

9 Lai MM, Luo HR, Burnett PE, et al. The calcineurin-binding protein cain is a negative regulator of synaptic vesicle endocytosis. J Biol Chem 2000;275:34017-20.

10 Lyson T, Ermel LD, Belshaw PJ, et al. Cyclosporine- and FK506-induced sympathetic activation correlates with calcineurin-mediated inhibition of T-cell signaling. Circ Res 1993;73:596-602.

11 Oliverio PJ, Lucas R, Mitchell SA, et al. Reversible tacrolimus-induced neurotoxicity isolated to the brain stem. Am J Neuroradiol 2000;21:1251-4.

12 Openshaw H, Slatkin NE, Smith E. Eye movement disorders in bone marrow transplant patients on cyclosporin and ganciclovir. Bone Marrow Transplant 1997; 19:503-5.

13 McMaster P, Mirza DF, Ismail T, et al. Therapeutic drug monitoring of tacrolimus in clinical transplantation. Ther Drug Monit 1995;17:602-5.

14 Selzner N, Durand F, Bernuau J, et al. Conversion from cyclosporine to FK506 in adult liver transplant recipients: a combined North American and European experience. Transplantation $2001 ; 72: 1061-5$ 